\title{
DETECTION OF SUSPICIOUS LESIONS IN DYNAMIC CONTRAST ENHANCED MRI DATA
}

\author{
T. Twellmann ${ }^{1}$, A. Saalbach ${ }^{1}$, C. Müller ${ }^{1}$, T. W. Nattkemper ${ }^{1}$, A.Wismüller ${ }^{2}$ \\ ${ }^{1}$ Applied Neuroinformatics Group, Faculty of Technology, Bielefeld University, Germany* \\ ${ }^{2}$ Department of Radiology, University of Munich, Germany
}

\begin{abstract}
Dynamic contrast-enhanced magnetic resonance imaging (DCEMRI) has become an important source of information to aid breast cancer diagnosis. Nevertheless, next to the temporal sequence of 3D volume data from the DCE-MRI technique, the radiologist commonly adducts information from other modalities for his final diagnosis. Thus, the diagnosis process is time consuming and tools are required to support the human expert. We investigate an automatic approach that detects the location and delineates the extent of suspicious masses in multi-temporal DCE-MRI data sets. It applies the state-of-the-art support vector machine algorithm to the classification of the short-time series associated with each voxel. The ROC analysis shows an increased specificity in contrast to standard evaluations techniques.
\end{abstract}

Keywords-Dynamic Contrast Enhanced MRI, Multi-temporal Image Analysis, Object Detection, Support Vector Machine

\section{INTRODUCTION}

In recent years, much effort has been spend to improve diagnosis techniques for breast cancer, one of the most common type of malignancy in industrialised nations. Recently, Dynamic contrastenhanced magnetic resonance imaging (DCE-MRI) has been proposed for breast cancer diagnosis. In contrast to the most commonly applied X-ray mammography technique, DCE-MRI provides the radiologist with 3D highly sensitive visualisations of the breast. Recording a short time series of $n_{t}$ volumes after applications of a gadolinium based contrast agent (Gd-DTPA) associates each voxel with spatial position $\mathbf{p}=(x, y, z)$ with a $n_{t}$-dimensional temporal signal $\mathbf{x}_{\mathbf{p}}$. Thus, each temporal sequence of DCE-MRI images can be regarded as a $4 \mathrm{D}$ volume with three spatial and one temporal dimension.

According to Kuhl et al. [1] the short-time series $\mathbf{x}_{\mathbf{p}}$ allow to infer valuable information about the local tissue states. Important criteria for suspicious series are e.g. the up-take speed of the contrast agent concentration or the presence of a contrast agent wash-out. The discriminative power of different kinetic and morphological diagnostic criteria for the time series has been recently investigated in more detail by Szabo et al. in [2].

Since the information about tissue states is distributed over the $n_{t}$ volumes, the locations of suspicious lesions can not be observed by one-on-one examination of single volumes but by examination of temporal changes between two or several different volumes of the sequence. To this end, experienced radiologists commonly screen the data by means of substraction images between volumes

*Contact: ttwellma@TechFak.Uni-Bielefeld.de at two different points of time. By exploration of suitable subtraction images, the characteristic up-take and wash-out of the contrast agent in cancerous lesions can be observed as a high intensity values while fat and muscle tissue remain dark. Since this method is time-consuming and only allows access to a small fraction of the available information, more sophisticated techniques such as the three point method [3] have been proposed which code a parametric description of the up-take and wash-out dynamic as a single colorised visualisation.

In the past, artificial neural networks (ANN) have been widely used in various fields of MRI data analysis in the first instance brain tumour segmentation [4] and recently also for analysing DCE-MRI data. In [5] Wismüller et al. applied an unsupervised ANN for the segmentation of DCE-MRI data. In [6] Lucht, Knopp and Brix applied a multilayer perceptron to the task of benign/malignant classification of the temporal signals. The data consisted of short-time series which were averaged over selected compartments of ROIs containing suspicious lesions marked by an expert using a visualisation based on a pharmacokinetic twocompartment model.

Typically, the radiologist's classification of the type of a suspicious lesion is based on the aggregated information derived from several modalities such as DCE-MRI, X-ray or sonography. As a consequence, the diagnostic process tends to be time consuming and tools which support the radiologist during the data screening are of special interest. Therefore, the task of automatic detection of suspicious lesions in DCE-MRI images is investigated in this work. Instead of finding single coordinates describing e.g. the coarse centres of mass of lesions, the goal is also to delineate the extent of the suspicious masses. To this end, the well-known learning algorithms Fisher's Linear Discriminant Analysis (FLDA) and Support Vector Machine (SVM) are applied for classification of the shorttime series $\mathbf{x}_{\mathbf{p}}$ as normal or suspicious signals. Assigning the classifiers output for signal $\mathbf{x}_{\mathbf{p}}$ to the corresponding positions $\mathbf{p}$ leads to a new $3 \mathrm{D}$ volume. This confidence volume shows masses of suspicious lesions as clusters of high confidence values and can be used to augment the visualisation of DCE-MRI data and to guide the radiologist's attention efficiently to positions of suspicious tissues. Since in general, the discriminative characteristic of the temporal kinetics of benign lesions is not as distinctive as for malignant lesions it is investigated furthermore whether the detection performance depends on the lesion type.

\section{ALGORITHMS}

The detection of suspicious lesion is formulated as a binary classification problem. Examples $\mathbf{x} \in \mathcal{D}$ from a data space $\mathcal{D}=\mathbb{R}^{n_{t}}$ 
have to be classified belonging to the class of normal signals (label $y=-1$ ) or to the class of suspicious signals (label $y=+1$ ). To this end, the algorithms are adapted using sets of training data $\Gamma=\Gamma_{N} \cup \Gamma_{S}=\left\{\left(\mathbf{x}_{i}, y_{i}\right)\right\}, \quad i=1, \ldots, l$ with the set $\Gamma_{S}$ of suspicious signals and the set of normal signals $\Gamma_{N}$.

\subsection{Fisher's Linear Discriminant Analysis}

Discrimination with the FLDA is based on the idea of finding a linear combination $\mathbf{w}$ of the features $\mathbf{x}=\left(x^{(1)}, \ldots, x^{\left(n_{t}\right)}\right)$ that maximises the ratio between the between-class scatter and the withinclass scatter described by the corresponding matrices $\mathbf{S}_{w}$, resp. $\mathbf{S}_{b}$ [7]. For the binary case, an analytical solution can be found which maximises the Rayleigh coefficient

$$
J(\mathbf{w})=\frac{\mathbf{w}^{T} \mathbf{S}_{b} \mathbf{w}}{\mathbf{w}^{T} \mathbf{S}_{w} \mathbf{w}}
$$

using

$$
\mathbf{w}=\mathbf{S}_{w}^{-1}\left(\mathbf{m}_{\Gamma_{S}}-\mathbf{m}_{\Gamma_{N}}\right)
$$

with class specific mean vectors $\mathbf{m}_{i}$. The fact that the algorithm has no further hyperparameters and that it is computational inexpensive even for huge data sets makes it especially appropriate for the given task. The binary classification for a example $\mathbf{x}_{j}$ is obtained by $\tilde{y}_{j}=\operatorname{sgn}\left[\left\langle\mathbf{w} \cdot \mathbf{x}_{j}\right\rangle+b\right]$ for a suitable bias value $b$.

\subsection{Support Vector Machine}

Kernel based methods such as SVMs have gained much attention from the machine learning and pattern recognition community in recent years. Founded on the Statistical Learning Theory [8], SVMs are currently the state-of-the-art algorithm for solving binary classification problems and have shown excellent results for various pattern recognition tasks [9]. For a given set of training data, SVMs find the discriminating hyperplane that separates the classes with maximal margin, i.e. maximal distance to the nearest examples, by optimising a quadratic program whose dual form is given by

$$
\begin{array}{cc}
\operatorname{maximize} & \sum_{i}^{l} \alpha_{i}-\frac{1}{2} \sum_{i, j}^{l} y_{i} y_{j} \alpha_{i} \alpha_{j}\left\langle\mathbf{x}_{i} \cdot \mathbf{x}_{j}\right\rangle \\
\text { w.r.t. } & \sum_{i}^{l} \alpha_{i} y_{i}=0 \\
& 0 \leq \alpha_{i} \leq C \quad i=1, \ldots, l
\end{array}
$$

Typically, most of the Lagrange coefficients $\alpha_{i}$ tend to be zero. Thus, the hyperplane

$$
\mathbf{w}=\sum_{i}^{l} \alpha_{i} y_{i} \mathbf{x}_{i}+b
$$

depends only on a small subset of the training data, the support vectors, and the bias $b$. By adjusting the regularisation parameter $C$ to suitable values, linear solutions can be found even for non-linear separable training data by tolerating a certain amount of classifications errors (soft-margin solution). For more complex discrimination functions, the inner product $\left\langle\mathbf{x}_{i} \cdot \mathbf{x}_{j}\right\rangle$ in (3) and in the discrimination function

$$
\tilde{y}_{j}=\operatorname{sgn}\left[\left\langle\mathbf{w} \cdot \mathbf{x}_{j}\right\rangle+b\right]=\operatorname{sgn}\left[\sum_{i}^{l} \alpha_{i} y_{i}\left\langle\mathbf{x}_{i} \cdot \mathbf{x}_{j}\right\rangle+b\right]
$$
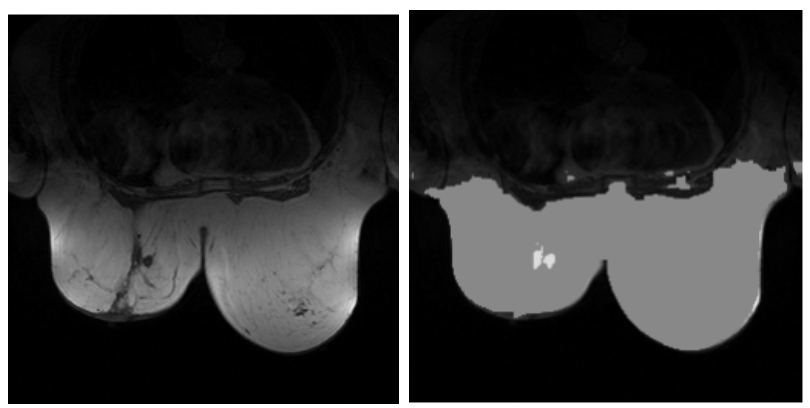

Fig. 1. Left: Slice of a pre-contrast image $(t=1)$. Right: Slice of corresponding breast mask (dark gray) with bifocal lesion (light gray).

can be replaced by non-linear kernel functions fulfilling the Mercer's theorem [10][11]. Thereby, the algorithm is executed in some feature space $\mathcal{F}$ which is non-linear related to the original data space and the maximal margin hyperplane in $\mathcal{F}$ corresponds to a non-linear discrimination function in $\mathcal{D}{ }^{1}$

\section{EXPERIMENT}

DCE-MRI was applied to six women with histologically proven malignant tumours and six women with proven benign lesions. These data were acquired according to an MRI protocol to investigate focal breast lesions.

The imaging process was performed with a 1,5 $\mathrm{T}$ system $^{2}$ equipped with a dedicated surface coil to enable simultaneous imaging of both breasts. First, transversal images were acquired with a STIR (short tau inversion recovery) sequence $(\mathrm{TR}=5600 \mathrm{~ms}$, $\mathrm{TE}=60 \mathrm{~ms}$, $\mathrm{FA}=90^{\circ}, \mathrm{TI}=150 \mathrm{~ms}$, matrix size of $256 \times 256$ pixels, slice thickness $4 \mathrm{~mm}$ ), then a dynamic T1 weighted gradient echo sequence (3D FLASH) was performed $\left(\mathrm{TR}=12 \mathrm{~ms}, \mathrm{TE}=5 \mathrm{~ms}, \mathrm{FA}=25^{\circ}\right)$ in transversal slice orientation with a matrix size $256 \times 256$ pixels and an effective slice thickness of $4 \mathrm{~mm}$. The dynamic study consisted of 6 measurements with an interval of $110 \mathrm{~s}$. The first frame was acquired before a bolus injection of a paramagnetic contrast agent $^{3}$, immediately followed by the other 5 measurements.

Each case was manually evaluated by an experienced radiologist who marked voxels of suspicious lesions using a cursor on a screening device. The lesion size varied between 26 to 743 voxels with an average size of 208 voxels.

\subsection{Preprocessing}

For each of the twelve cases, a mask for the region of potential breast tumours is extracted using the following automatic thresholding method $^{4}$ : After application of a $3 \times 3 \times 3$ median filter to the

\footnotetext{
${ }^{1}$ For the presented setup, preliminary experiments with the SVM combined with the non-linear Gaussian Kernel have shown no superior performance in contrast to the linear SVM using the inner product as a kernel. Therefore, only the linear SVM is considered in following experiments.

${ }^{2}$ Magneton Vision, Siemens, Erlangen, Germany

${ }^{3}$ Magnevist, Schering, Berlin, Germany

${ }^{4}$ The evaluations in the following experiment are restricted to the masked subregions since they reflect the areas where a radiologist would expect breast cancer lesions. Thus restricting the ROC analysis to this region gives a more realistic performance measures since e.g. the heart which commonly causes a high number of false positives is excluded.
} 
pre-contrast image, a threshold is calculated from the image's bimodal shaped histogram which separates voxels of the bright areas of muscle and fat tissues in the breast from voxels in the darker areas of the thorax and background. The threshold is automatically determined using the method presented in [12]. Application of a $5 \times 5 \times 5$ closing operator on the thresholded image assures a closed mask (referred to as breast mask) for the breast area (Fig.1). The only preprocessing at the level of individual signal time curves $\mathbf{x}_{\mathbf{p}}$ is a normalisation with respect to the corresponding pre-contrast value $x_{\mathbf{p}}^{(1)}$ :

$$
\mathbf{x}_{\mathbf{p}}=\left(x_{\mathbf{p}}^{(2)}-x_{\mathbf{p}}^{(1)}, \ldots, x_{\mathbf{p}}^{\left(n_{t}\right)}-x_{\mathbf{p}}^{(1)}\right) .
$$

\subsection{Training}

Since the FLDA is computational inexpensive, $\Gamma_{N}$ consists of all examples from the breast mask, except those inside the lesion masked by the radiologist which are taken for $\Gamma_{S}$.

Even though very efficient methods have been proposed for the SVM algorithm [13], training with all available data is impractical because of two reasons: $(i)$ The computational expense would be untraceable for data sets of about $3 \cdot 10^{6}$ examples. (ii) Using all data would cause an extreme unbalance between number of tumour signals (about $2.5 \cdot 10^{3}$ total) and the number of normal tissue voxels which commonly degenerates the performance of learning algorithms. Thus, only a random subset of the normal signals is used for the training. Further more, each component of the examples is scaled to a range of $[-1, \ldots,+1]$ for numerical reasons.

For selecting the optimal parametrisation for SVM, the data is divided in a training set and a test set. The training set consisted of $66 \%$ of the suspicious signals and a random subset of normal signals. The ratio $r$ between number of suspicious and normal signals in the training data is adjusted during the parameter optimisation. The test set is assembled from the remaining $34 \%$ of the suspicious signals and a random subset of normal signals with a constant ratio of $1 / 200$. The regularisation parameter $C$ and the ratio $r$ are selected by estimating the performance on the test set for different tuples $(C, r), C=10^{k}, k \in\{0,0.44,0.88, \ldots, 4.0\}, r \in$ $\left\{\frac{1}{1}, \frac{1}{3}, \frac{1}{5}\right\}$.

The validation case is evaluated with the parameter tuple yielding the maximal area under the ROC curve (AUC) [14] value for the test set. To lessen the influence of the random sampling of normal signals, the procedure is repeated ten times for each validation case in order to average the performance values.

\subsection{Evaluation}

In order to give realistic estimates of the detection performance, both learning algorithms are evaluated using a leave-one-case-out scheme. In the first setup, the algorithms are adapted using signals solely taken from eleven of the twelve cases. After training and parameter optimisation, the discrimination performance is estimated on the twelfth unseen validation case. The scheme is repeated twelve times, each time excluding a different case (Setup I).

In order to examine dependencies of the detection performance on the lesion type, the above scheme is restricted to cases with the same lesion type in a second setup. After training with data taken solely from five malignant/benign cases, the algorithms are applied to the excluded sixth malignant/benign validation case. Again, this scheme is repeated six times using one of the six cases for validation (Setup II).
Table 1. AUC (top) and $\mathrm{AUC}_{\mathrm{SE} \geq 0.9}$ (bottom) for setup I.

\begin{tabular}{|c||c|c|c|}
\hline \multicolumn{4}{|c|}{ AUC } \\
\hline Case & SI & FLDA & SVM \\
\hline \hline$m_{A l l}$ & $0.980 \pm 0.023$ & $0.987 \pm 0.013$ & $0.989 \pm 0.012$ \\
$m_{\mathcal{M}}$ & $0.974 \pm 0.029$ & $0.983 \pm 0.016$ & $0.984 \pm 0.016$ \\
$m_{\mathcal{B}}$ & $0.985 \pm 0.015$ & $0.991 \pm 0.007$ & $0.993 \pm 0.005$ \\
\hline
\end{tabular}

\begin{tabular}{|c||c|c|c|}
\hline \multicolumn{4}{|c|}{$\mathrm{AUC}_{\mathrm{SE} \geq 0.9}$} \\
\hline Case & SI & FLDA & SVM \\
\hline \hline$m_{\text {All }}$ & $0.895 \pm 0.112$ & $0.924 \pm 0.067$ & $0.946 \pm 0.053$ \\
$m_{\mathcal{M}}$ & $0.872 \pm 0.137$ & $0.907 \pm 0.081$ & $0.928 \pm 0.066$ \\
$m_{\mathcal{B}}$ & $0.918 \pm 0.087$ & $0.940 \pm 0.050$ & $0.964 \pm 0.031$ \\
\hline
\end{tabular}

In accordance to common practice, the detection performance is analysed by ROC analysis which is independent from a specific choice of threshold values. Thus for each validation case, the AUC value are calculated using the projection $c_{\mathbf{p}}=\left\langle\mathbf{w} \cdot \mathbf{x}_{\mathbf{p}}\right\rangle$ of the example $\mathbf{x}_{\mathbf{p}}$ on the weight vector $\mathbf{w}$ of the FLDA/SVM hyperplane as the confidence that $\mathbf{x}_{\mathbf{p}}$ belongs to the class of suspicious signals. As a second performance measure, referred to as $\mathrm{AUC}_{\mathrm{SE} \geq 0.9}$, the area under the ROC curve above a minimum sensitivity of 0.9 is used which is motivated by our particular interest in the extent of the lesion. For comparison reasons, the measure is normalised to $[0.0, \ldots, 1.0]$. The results are also compared to the AUC and AUC $\mathrm{SE}_{\mathrm{SE}} \geq 0.9$ values yielded by a simple subtraction image (SI). In this case, the confidence value is the difference between the voxel intensity in the second post-contrast image $(t=3)$ and in the pre-contrast image $(t=1) c_{\mathbf{p}}=x_{\mathbf{p}}^{(3)}-x_{\mathbf{p}}^{(1)}$ which is primarily sensitive to the fast uptake of the contrast agent concentration in suspicious lesions.

\section{RESULTS}

Table 1 shows the AUC and $\mathrm{AUC}_{\mathrm{SE} \geq 0.9}$ values for setup I averaged over all twelve cases (row $m_{A l l}$ ). In row $m_{\mathcal{M}}$ and $m_{\mathcal{B}}$ the detection performance of the first setup is shown in more detail by averaging the $\mathrm{AUC}$ and $\mathrm{AUC}_{\mathrm{SE}} \geq 0.9$ solely over six malignant cases $\left(m_{\mathcal{M}}\right)$, resp. over six benign cases $\left(m_{\mathcal{B}}\right)$.

Regarding the AUC values all three methods show comparable excellent performance. Nevertheless, a paired $t$-test indicates that the difference between SI and SVM is significant ${ }^{5}$. Since the major fraction of the region covered by the breast mask contains fat and muscle tissue, which has an easy to discriminate temporal signal characteristic, the AUC value is exceptional high. The major fraction of false positives are caused by blood vessels, in the region of the ribcage and by small registration errors at the skin as one can see by mean of high confidence values in Fig. 2. Considering the $\mathrm{AUC}_{\mathrm{SE} \geq 0.9}$ values, the superior performance of the learning algorithms becomes more evident. In this case, the SVM significantly ${ }^{6}$ outperforms SI and is slightly superior to the FLDA. Further more, the lower standard deviations of the SVM indicates a more stable performance. The benefit of the SVM becomes also apparent if a threshold is adjusted to a sensitivity of approx. 0.9 for each case. For ten cases the SVM shows an average reduction of false positives by $65 \%$ in contrast to the SI. Only for two cases, the number increases by about $220 \%$.

\footnotetext{
${ }^{5}$ two-tailed $p=0.0492, t(11)=2.21, C I=95 \%$

${ }^{6}$ paired $t$-test: two-tailed $p=0.034, t(11)=2.42, C I=95 \%$
} 

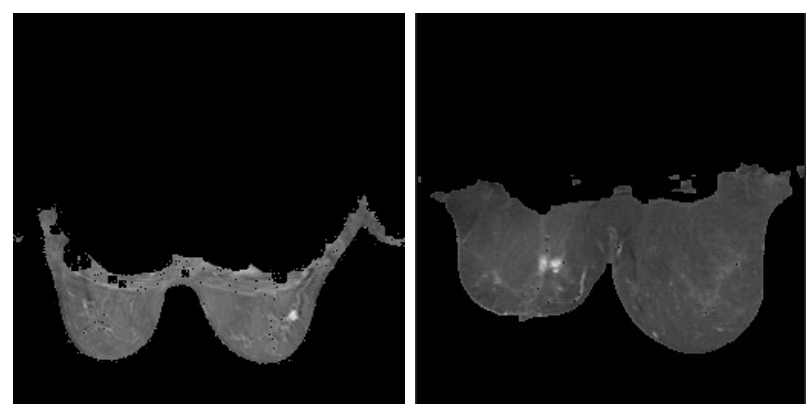

Fig. 2. Slice of two confidence volumes based on the SVM. The values are scaled to the range $[0, \ldots, 255]$ for the purpose of visualisation.

Table 2. $\mathrm{AUC}$ (top) and $\mathrm{AUC}_{\mathrm{SE} \geq 0.9}$ (bottom) values for setup II.

\begin{tabular}{|c|c|c|c|}
\hline \multicolumn{4}{|c|}{ AUC } \\
\hline Case & SI & FLDA & SVM \\
\hline$m_{\mathcal{M}}$ & $0.974 \pm 0.029$ & $0.980 \pm 0.019$ & $0.982 \pm 0.019$ \\
\hline$m_{\mathcal{B}}$ & $0.985 \pm 0.015$ & $0.993 \pm 0.005$ & $0.994 \pm 0.004$ \\
\hline \multicolumn{4}{|c|}{$\mathrm{AUC}_{\mathrm{SE}>0.9}$} \\
\hline Case & SI & FLDA & SVM \\
\hline$m_{\mathcal{M}}$ & $0.872 \pm 0.137$ & $0.885 \pm 0.111$ & $0.908 \pm 0.088$ \\
\hline$m_{\mathcal{B}}$ & $0.918 \pm 0.087$ & $0.962 \pm 0.029$ & $0.970 \pm 0.022$ \\
\hline
\end{tabular}

One would expect that malignant lesions are easier to detect than benign lesions because of their characteristic fast uptake followed by a washout of the contrast agent. Instead, the average performance for benign cases $m_{\mathcal{B}}$ is greater than for malignant cases $m_{\mathcal{M}}$ for both measures. The increased performance for benign cases becomes more obviously in the results for setup II. Especially the $\mathrm{AUC}_{\mathrm{SE} \geq 0.9}$ in Tab. 2 indicates an increased detection performance with a lower standard deviation for benign cases. One possible reason could be the heterogeneity of the set of time series from malignant lesions. Even if these lesions are marked as malignant, a significant fractions of it's short-time series commonly show benign characteristics. In contrast, a more compact data distribution can be assumed for the set of signals from benign labeled lesions. Thus, the benign signals in the training data of setup II also improve the detection performance for malignant lesions which is also expressed by the decreasing $\mathrm{AUC}_{\mathrm{SE} \geq 0.9}$ values for $m_{\mathcal{M}}$ comparing Tab.1 and Tab.2.

\section{CONCLUSION}

We have proposed a machine learning approach for improved detection of suspicious lesions based on classification of single short time series. Compared to subtraction images which capture only a fraction of the information of DCE-MRI data, our approach exploits the complete information of the $n_{t}$-dimensional signals and is able reduce the number of false positives further on with a constant high sensitivity. Thus, even small benign and malignant masses can be localised by evaluation of the confidence volumes which are calculated in less than 2 minutes due to the computational simplicity of the hyperplane classifiers.

Since the training of the system is based on data-driven procedure, it does not depend on an explicit signal model and therefore can be easily adapted to varying imaging protocols and to new data domains.

\section{REFERENCES}

[1] C. K. Kuhl, P. Mielcareck, S. Klaschik, C. Leutner, E. Wardelmann, J. Gieseke, and H. H. Schild, "Dynamic breast MR imaging: Are signal intensity time course data useful for differential diagnosis of enhancing lesions?," Radiology, vol. 211, no. 101, 1999.

[2] B. K. Szabo, P. Aspelin, M. Kristoffersen Wiberg, and B. Bone, "Dynamic MR Imaging of the Breast - Analysis of kinetic and morphologic diagnostic criteria," Acta Radiologica, vol. 44, pp. 379-386, 2003.

[3] D. Weinstein, S. Strano, P. Cohen, S. Fields, J. M. Gomori, and H. Degani, "Mapping pathophysiological features if breast fibroadenoma by the three time point (3tp) contrast enhanced MRI method: pilot study," Radiology, vol. 210, pp. 233-240, 1999.

[4] L. P. Clarke, R. P. Velthuizen, M. A. Camacho, J. J. Heine, M. Vaidyanathan, L. O. Hall, R. W. Thatcher, and M. L. Silbiger, "MRI Segmentation: Methods and Applications," Magnetic Resonance Imaging, vol. 13, no. 3, pp. 343-368, 1995.

[5] A.Wismüller, O. Lange, D. Dersch, G. Leinsinger, K .Hahn, B. Ptz, and D. Auer, "Cluster analysis of biomedical image time series," Int. Journal of Computer Vision, vol. 46, no. 2, pp. 103-128, 2002.

[6] R. E. A. Lucht, M. V. Knopp, and G. Brix, "Classification of signal-time curves from dynamic MR mammography by neural networks," Magnetic Resonance Imaging, vol. 19, pp. 51-57, 2001.

[7] R. O. Duda and P. E. Hart, Pattern Classification and Scene Analysis, Wiley, 1973.

[8] V. Vapnik, The nature of statistical learning theory, Springer, 1995.

[9] K. P. Bennett and C. Campbell, "Support vector machines: hype or hallelujah?," SIGKDD Explor. Newsl., vol. 2, no. 2, pp. 1-13, 2000.

[10] B. Schölkopf and A. Smola, Learning with Kernels, MIT Press, 2002.

[11] B. Schölkopf, S. Mika., C. Burges, Knirsch, K. R. Müller, G. Rätsch, and A. J. Smola, "Input space vs. feature space in kernel-based methods," IEEE Trans. on Neural Networks, vol. 10, no. 5, pp. 1000-1017, 1999.

[12] N. Otsu, "A threshold selection method from gray level histograms," IEEE Trans. Systems, Man and Cybernetics, vol. 9, no. 1, pp. 62-66, 1979.

[13] J. Platt, "Advances in Kernel Methods - Support Vector Learning," chapter Fast training of support vector machines using sequential minimal optimization, pp. 185-208. MIT Press, 1999.

[14] J. A. Hanley, "Receiver operating characteristic methodology: the state of the art," CRC critical reviews in diagnostic imaging, vol. 29, pp. 307-335, 1989. 